\title{
Evaluation De La Denutrition Chez Les Enfants Ages De 2 A 60 Mois Hospitalises Aux Services De Pediatrie Des Hopitaux De Ziguinchor (Senegal)
}

\author{
Lamine Thiam, \\ Hopital de la paix de Ziguinchor, Université Assane Seck de Ziguinchor \\ Babacar Niang, \\ Hopital d'enfants Albert Royer de Dakar, \\ Université Cheikh Anta Diop de Dakar \\ Francois Niokhor Diouf, \\ Hopital régional de Ziguinchor \\ Isabelle Jokébé Coly, \\ Assane Dramé, \\ Rozi Tchiou Issa, \\ Hopital de la paix de Ziguinchor \\ Saliou Diouf, \\ Hopital d'enfants Albert Royer de Dakar, \\ Université Cheikh Anta Diop de Dakar \\ Assane Sylla,
}

Hopital Ledantec de Dakar, Université Cheikh Anta Diop de Dakar

Amadou Lamine Fall,

Ousmane Ndiaye,

Hopital d'enfants Albert Royer de Dakar,

Université Cheikh Anta Diop de Dakar

Doi: 10.19044/esj.2018.v14n3p452 URL:http://dx.doi.org/10.19044/esj.2018.v14n3p452

\begin{abstract}
Introduction: Malnutrition is a public health problem in developing countries. The prevalence of malnutrition at the national level hardly reflects the reality in hospitals. It is in this context that we assessed the nutritional status of children from 2 months to 5 years hospitalized in the pediatric ward of the regional hospital and the Ziguinchor Peace Hospital. Materials and methods: This was a prospective study from June 1 to October 30, 2016. Children aged 2 to 60 months, hospitalized in one of the two services, were included. Children with esdato-ascetic syndrome were not included. Epidemiological, anthropometric data, and associated diagnosis were studied.
\end{abstract}


Results: We included 114 children (70 boys and 44 girls). The average age was 21.9 months [4-59]. Forty-two point one percent (42.1\%) of infants $<6$ months were breastfed exclusively with breast milk. The weaning of children was done early in $55.3 \%$. The mean age of mothers was 26.6 years [17-38]. The socioeconomic level was low in $62.3 \%$ of cases. The prevalence of malnutrition averaged $35.5 \%$ for underweight; $32.9 \%$ for wasting and $32.0 \%$ for stunting. Acute respiratory infections (ARI) and acute gastroenteritis were the most common associated conditions. Mean hospital stay was 8 days \pm 009 [3-28]. About two thirds of the patients $(n=72)$ had a hospital stay of more than 7 days. Malnutrition was significantly associated with multiparity, low socioeconomic status, hospital stay of more than 7 days and infectious diseases. Conclusion: Infections and a hospital stay longer than a week are factors favoring malnutrition in children from 2 months to 5 years.

Keywords: Malnutrition, child under 5 years and hospitalization

\section{Resume}

Introduction : La malnutrition représente un problème de santé publique dans les pays en développement. La prévalence de la malnutrition au niveau national reflète peu la réalité en milieu hospitalier. C'est dans ce contexte que nous avons évalué l'état nutritionnel des enfants de 2 mois à 5 ans hospitalisés dans les services de pédiatrie du centre hospitalier régional et de l'hôpital de la paix de Ziguinchor. Matériel et méthodes : Il s'agissait d'une étude prospective allant du 1er juin au 30 octobre 2016. Ont été inclus les enfants âgés de 2 à 60 mois, hospitalisés dans l'un des deux services. N'ont pas été inclus les enfants présentant un syndrome œdémato-ascétique. Les données épidémiologiques, anthropométriques, et le diagnostic associé ont été étudiés. Résultats : Nous avons inclus 114 enfants (70 garçons et 44 filles). L'âge moyen était de 21,9 mois [4-59]. Quarante-deux virgule un pour cent $(42,1 \%)$ des nourrissons âgés de moins de 6 mois, étaient allaités exclusivement au lait maternel. Le sevrage des enfants était fait précocement dans 55,3\%. L'âge moyen des mères était de 26,6 ans [17-38]. Le niveau socioéconomique était bas dans $62,3 \%$ des cas. La prévalence de la malnutrition était en moyenne de 35,5\% pour l'insuffisance pondérale ; $32,9 \%$ pour l'émaciation et $32,0 \%$ pour le retard de croissance. Les infections respiratoires aigües (IRA) et la gastroentérite aigue étaient les pathologies associées les plus fréquentes. La durée d'hospitalisation moyenne était de 8 jours \pm 009 [3-28]. Environ deux tiers des malades $(n=72)$ avaient une durée d'hospitalisation de plus de 7 jours. La malnutrition était significativement associée à la multiparité, à un niveau socioéconomique bas, à un séjour hospitalier de plus de 7 jours et à des pathologies infectieuses. Conclusion : Les infections et une durée d'hospitalisation supérieure à une semaine 
constituent des facteurs favorisant de la malnutrition chez les enfants de 2 mois à 5 ans.

Mots-Clés : Malnutrition, enfant de moins de 5 ans et hospitalisation

\section{Introduction}

La malnutrition est un état pathologique résultant de la carence ou de l'excès relatif ou absolu d'un ou de plusieurs nutriments essentiels. Elle pose un problème de santé publique dans le monde et surtout dans les pays en développement (Mali, 2002).

Au Sénégal 1'EDS-C de 2015 notait un taux de $21 \%$ de malnutrition chronique et un taux de $8 \%$ de malnutrition aigüe chez les enfants de moins de 5 ans. Cette prévalence de la malnutrition dans cette tranche âge de la population générale ne reflète pas la situation en milieu hospitalier ((EDSContinue Sénégal, 2015) (Dillon, 2003)). Généralement aucune mesure préventive n'est prise lors de l'hospitalisation pour diverses affections qui exposent à un risque de dénutrition. Les patients sont rarement évalués sur le plan nutritionnel à l'admission, et les équipes soignantes n'affichent que sporadiquement une vigilance sur le régime diététique des patients.

C'est ainsi que nous avons mené cette étude dont l'objectif principal était d'évaluer l'état nutritionnel des enfants hospitalisés dans les services de pédiatries des hôpitaux de Ziguinchor (hôpital de la paix et hôpital régional).

\section{Materiel et methode}

\section{Cadre d'étude}

L'étude s'est déroulée dans les services de pédiatrie de l'hôpital de la paix et du centre hospitalier régional de Ziguinchor.

Le centre hospitalier régional est une structure hospitalière de niveau 2. Le service de pédiatrie est dirigé par un pédiatre praticien hospitalier, assisté par une équipe d'infirmiers. Il a une capacité de 40 lits.

L'hôpital de la Paix de Ziguinchor est une nouvelle structure hospitalouniversitaire. Il comporte des services de médecine et de chirurgie et un pôle mère-enfant.

Le service de pédiatrie du pôle mère-enfant comporte une unité de néonatologie ; une unité d'hospitalisation pour grand enfant et un centre de récupération et d'éducation nutritionnelle (CREN). Il a une capacité de 30 lits et est dirigé par un pédiatre assistant universitaire, assisté par deux autres pédiatres praticiens hospitaliers.

Le CREN est spécialisé dans la prise en charge des enfants malnutris. Il dispose d'une grande salle servant d'accueil et d'évaluation nutritionnelle ; d'une cuisine et d'un aire de jeux. Il est dirigé par une seule infirmière d'état, assistée par les autres membres de l'équipe de la pédiatrie. 
Les deux services de pédiatrie reçoivent des nouveaux et des enfants de âgés moins de 15 ans venant de la région de Ziguinchor mais également de la sous-région (Kolda, Sédhiou, Gambie, Guinée).

\section{Type et période de l'étude}

Nous avons mené une étude transversale et descriptive qui s'est déroulée durant la période allant du lér juin au 30 octobre 2016.

\section{Population de l'étude}

Critères d'inclusion : étaient inclus dans l'étude tous les enfants âgés de 2 mois à 60 mois révolus; hospitalisés pendant plus de 48 heures. Les mesures anthropométriques ont été réalisées à l'entrée (avant la 48éme heure d'hospitalisation) et à la sortie.

Critères de non inclusion : n'ont pas été inclus dans l'étude les enfants hospitalisés pour une glomérulonéphrite, une cardiopathie ou une hépatopathie chronique.

\section{Collecte et paramètres étudiés}

Les paramètres étudiés étaient les données épidémiologiques, sociodémographiques et anthropométriques (poids, taille, périmètre brachial, périmètre thoracique). Le diagnostic retenu, la durée d'hospitalisation et le devenir du malade ont été également étudiés.

\section{Définition des paramètres}

Selon les normes de l'OMS de 2000, l'insuffisance pondérale était définit par l'indicateur poids (âge) < -2ET; le retard de croissance par l'indicateur taille (âge) $<-2 \mathrm{ET}$ et l'émaciation par l'indice poids (taille) $<$ 2ET.

\section{Analyse et saisie des données}

Le traitement des données a été effectué grâce au logiciel Epi Info. L'analyse statistique des relations a été effectuée grâce au test khi-deux et de Fischer. Une valeur de $\mathrm{p}<0,05$ a été considérée comme significative.

\section{Resultats}

Nous avons inclus au total 114 enfants ( 70 garçons et 44 filles) durant la période de l'étude. L'âge moyen était de 21,9 mois avec des extrêmes allant de 4 à 59 mois. Environ 1/4 (29) des patients venaient des régions comme Sédhiou, Kolda et de la sous-région (Guinée Bissau, Gambie...). Cinquantehuit virgule huit pour cent $(58,8 \%)$ des enfants étaient vaccinés selon le Programme Elargie de vaccination (PEV) du Sénégal. Quatre-vingt-quinze virgule six pour cent $(95,6 \%)$ des nourrissons de moins de 6 mois étaient allaités par le lait maternel et $42,1 \%$ parmi eux étaient exclusivement sous lait 
maternel. Plus de la moitié ( $\mathrm{n}=66$ soit $57,9 \%$ ) des enfants ont eu une alimentation diversifiée. Cette alimentation était précoce dans $46,5 \%$ et tardive dans $11,4 \%$. Le sevrage était fait précocement dans 55,3\%. L'âge moyen des mères était de 26,6 ans avec des extrêmes allant de 17 à 38 ans. La moitié des mères n'était pas scolarisée et plus de la moitié des familles $(65 \%)$ avait un bas niveau socio-économique. La répartition des enfants selon les indicateurs poids (âge) ; taille (âge) et poids (taille) était superposable entre l'admission et la sortie de l'hôpital (figures 1, 2, 3). La prévalence de la malnutrition dans notre étude était en moyenne de 35,5\% pour l'insuffisance pondérale ; 32,9\% pour l'émaciation et $32,0 \%$ pour le retard de croissance. Le tableau I donne la répartition des enfants selon le diagnostic associé. La durée d'hospitalisation moyenne était de 8 jours \pm 009 avec des extrêmes allant de 3 à 28 jours. Environ deux tiers des malades $(\mathrm{n}=72)$ avaient une durée d'hospitalisation de plus de 7 jours.

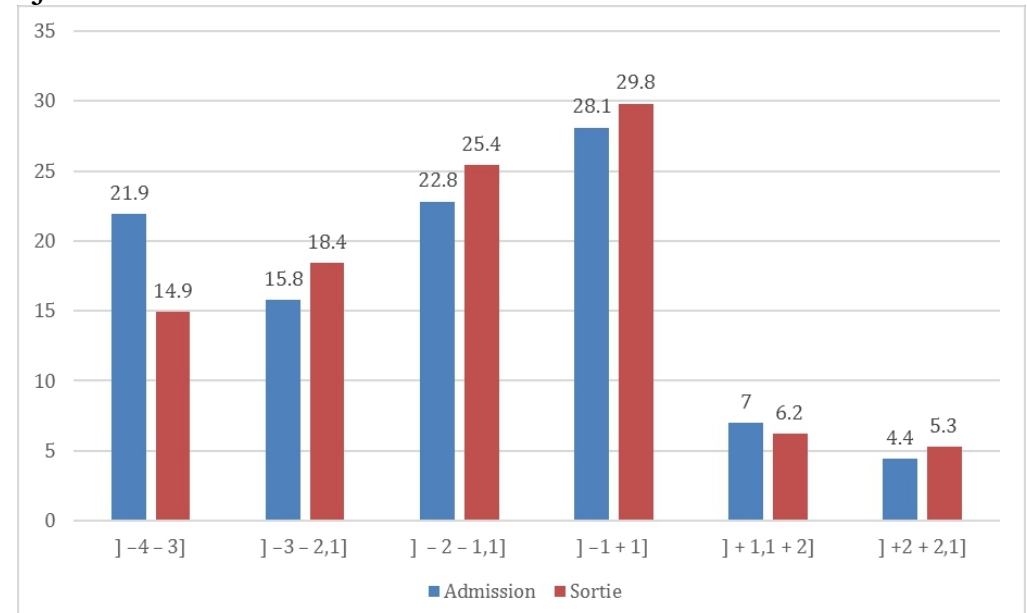

Figure 1 : répartition des enfants selon l'indicateur poids (âge) à l'admission et à la sortie

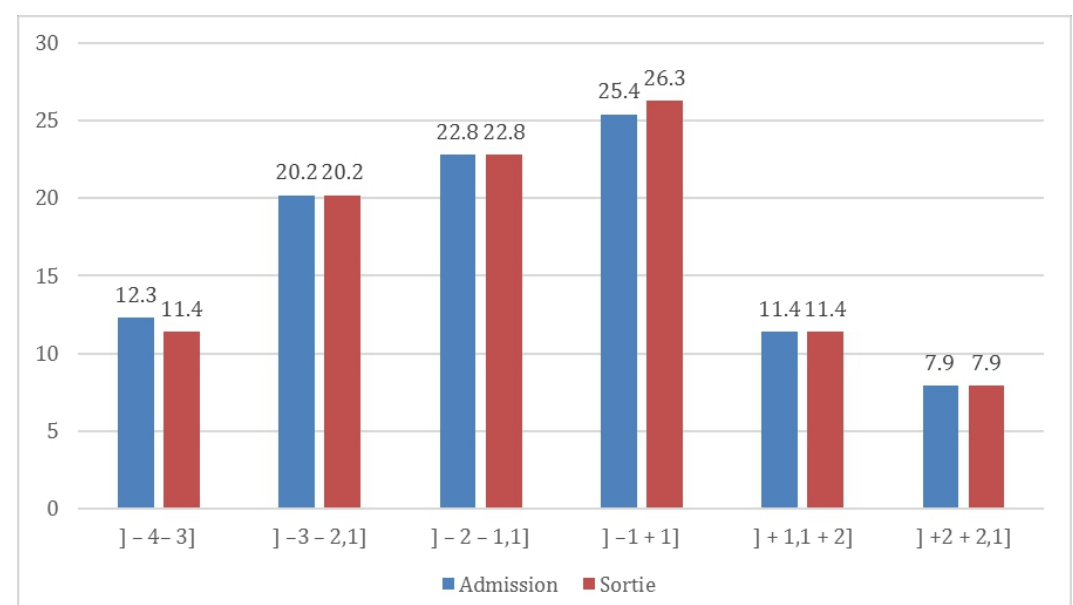

Figure 2 : répartition des enfants selon l'indicateur taille (âge) à l'admission et à la sortie 


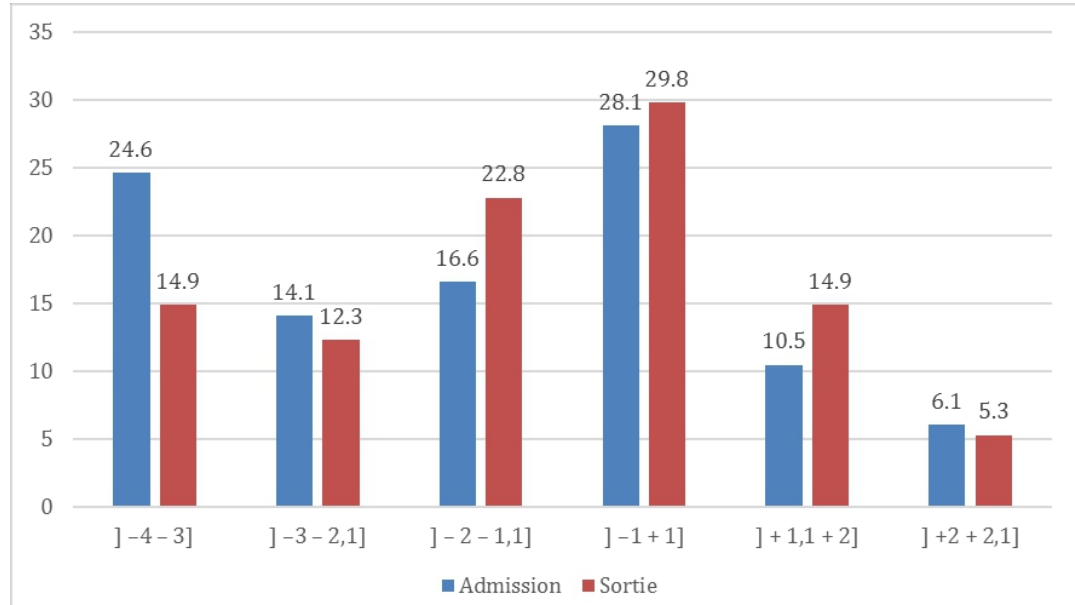

Figure 3 : répartition des enfants selon l'indice poids (taille) à l'admission et à la sortie

Tableau I : répartition des enfants selon le diagnostic associé

\begin{tabular}{|ccc|}
\hline Diagnostic retenu & Nombre & Pourcentage \\
\hline Gastro-entérite aigue fébrile & 34 & 29,8 \\
\hline Bronchiolite aigue surinfectée & 18 & 15,8 \\
\hline Pneumonie aigue & 16 & 14,1 \\
\hline Méningite bactérienne et viral & 14 & 12,3 \\
\hline Paludisme grave & 12 & 10,5 \\
\hline Staphylococcie pleuro-pulmonaire & 4 & 3,5 \\
\hline Tuberculose pulmonaire & 3 & 2,6 \\
\hline Tuberculose ganglionnaire & 2 & 1,7 \\
\hline Crise vaso-occlusive & 2 & 1,7 \\
\hline Infection à VIH & 2 & 1,7 \\
\hline Rhumatisme Articulaire Aigue & 1 & 0,9 \\
\hline Choc septique & 1 & 0,9 \\
\hline Epilepsie & 1 & 0,9 \\
\hline
\end{tabular}

Trois décès $(2,6 \%)$ ont été déplorés dans notre étude. Tous les trois (3) décès étaient survenus chez les enfants présentant une malnutrition aiguë sévère dont les deux (2) étaient associé à la tuberculose pulmonaire et un (1) à l'infection à VIH.

Dans notre étude, la malnutrition aigüe et la malnutrition chronique étaient associées de façon significative à la multiparité ( $\mathrm{p}$ value respectif à 0,035 et à 0,026 ) ; à un niveau socioéconomique bas de la famille ( $\mathrm{p}$ value respectif à 0,023 et à 0,014 ); aux pathologies infectieuses comme les Infection Respiratoires Aigües (IRA) et la Gastro-entérite Aigue (GEA) ( $p$ value respectif à 0,035 et à 0,012 ) et à un séjour de plus d'une semaine en milieu hospitalier ( $\mathrm{p}$ value respectif à 0,035 et à 0,043 )

\section{Discussion}

En utilisant les mêmes normes de l'OMS que dans l'EDS du Sénégal de 2015, les taux de malnutrition que nous avons retrouvés dans notre série 
sont plus élevés que ceux retrouvés au niveau national. Il y avait quatre fois plus d'émaciation dans notre série (32,9\%) qu'au niveau national $(8 \%)$; et 1,5 fois plus de retard de croissance dans notre série $(32,0 \%)$ qu'au niveau national $(21 \%)$.

Ces taux peuvent s'expliquer par le fait que notre étude concernait des enfants hospitalisés. Des résultats similaires ont été retrouvés par d'autres auteurs sénégalais auparavant ((Diouf, 1990) (Sarr/Sy, 2014)). Dans notre étude, les facteurs associés à la malnutrition étaient classiques (le bas niveau socioéconomique, le séjour prolongé à l'hôpital, la multiparité, la diversification et le sevrage précoces). Ces facteurs sont retrouvés dans plusieurs autres études sénégalaises et africaines ((Diouf, 1990) (Aouehougon, 2007) (EDS Cameroun, 2012) (EDS Sénégal, 2011) (Sibetcheu, 2005)). Une mauvaise conduite de l'alimentation entraine une malnutrition chez l'enfant. Dans notre étude, le taux d'allaitement maternel exclusif était de 42,1\%. Ce taux est supérieur à celui au niveau national (33\% selon l'EDS-C 2015). De façon générale, la faisabilité de l'allaitement maternel exclusif constitue un problème dans les pays en de développement et dans les pays développés. Pour améliorer le taux d'allaitement maternel exclusif, l'OMS et l'UNICEF ont mis en place de nouvelles directives à travers l'ANJE (Alimentation du nourrisson et du jeune enfant). L'objectif étant d'atteindre au moins 50\% en 2025.

\section{Conclusion}

La malnutrition chez les enfants de moins de 5 ans est plus fréquente dans nos services de pédiatrie par comparaison aux données nationales. Elle est associée au bas niveau socioéconomique des familles, à une durée d'hospitalisation de plus de 7 jours, à la multiparité chez la mère, à une diversification et un sevrage précoces de l'enfant.

Nous recommandons aux personnels de santé de la région de continuer à promouvoir l'allaitement maternel exclusif chez les nourrissons âgés de moins de 6 mois et d'évaluer l'état nutritionnel des enfants dès l'admission. Ils doivent également continuer à donner des repas et encourager activement les enfants à manger durant la période de l'hospitalisation.

\section{References:}

1. Cadre stratégique de lutte contre la pauvreté rapport final adopte par le gouvernement du Mali en Mai 2002.

2. Agence Nationale de la Statistique et de la Démographie (ANSD) [Sénégal], et ICF International. 2016. Sénégal : Enquête Démographique et de Santé Continue (EDS-Continue 2015). Rockville, Maryland, USA : ANSD et ICF International.

3. Dillon, J. C. Evolution de la santé des enfants dans le monde au cours de la période 1990 - 2000. Med Trop. 2003; 63: 407-12. 
4. Diouf, S., Diallo, A., Camara, B., Diagne, I., Tall, A., Sy, S. et al. La malnutrition proteino-calorique chez les enfants de moins 5 ans en zone rurale sénégalaise (khombole). Med Afr Noire. 1990; 37: 29-32.

5. Sarr/Sy, N. F. Evaluation nutritionnelle et sanitaire des enfants âgés de 0 à 23 mois à Kédougou et à Vélingara. [Thèse Med]. Dakar : UCAD ; 2014. $\mathrm{N}^{\circ} 38$.

6. Aouehougon, O. La malnutrition proteino-énergétique et ses facteurs de risque chez les enfants de moins de 5 ans dans le district sanitaire de Tougan [Mémoire Diplôme d'attaché de santé en épidémiologie]. Burkina Faso: Ecole Nationale de Santé Publique; 2007.

7. CAMEROUN. Ministère de la sante publique. Institut National de la Statistique (INS) et ICF. International. 2012. Enquête Démographique et de Santé et à indicateurs Multiples du Cameroun 2011. Calverton, Maryland, USA : INS et ICF International.

8. SENEGAL, Ministère de la Santé et de la Prévention Médicale. Enquête Démographique et de santé à Indicateurs Multiples au Sénégal 2010-11(EDS-MICS). Dakar, Juin 2011.

9. Sibetcheu, D., Fomo, M. A., Libite, P. R., Jazet, E. Enquête Démographique et de Santé et à indicateurs multiples du Cameroun 2011 (EDSC-MICS) Vol. 2. Calverton, Maryland, USA: INS et ORC Macro; 2005. Jun, Allaitement maternel, état nutritionnel des enfants et des femmes; pp. 179-207. 